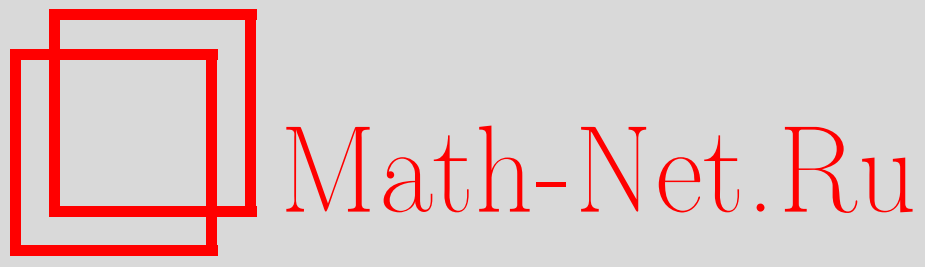

С. С. Акбаров, Свойство стереотипной аппроксимации и проблема однозначности следа, Функи. анализ и его прил., 1999, том 33, выпуск 2, 68-73

DOI: https://doi.org/10.4213/faa355

Использование Общероссийского математического портала MathNet.Ru подразумевает, что вы прочитали и согласны с пользовательским соглашением

http: //www.mathnet.ru/rus/agreement

Параметры загрузки:

IP : 107.22 .136 .117

26 апреля 2023 г., 10:13:28

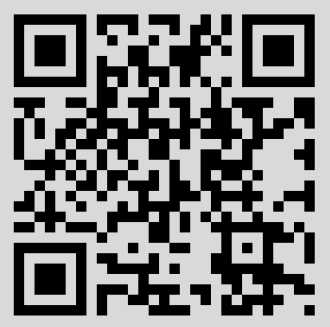




\title{
Свойство стереотипной аппроксимации и проблема однозначности следа
}

\author{
(c) 1999. C. С. Акьаров
}

В фундаментальной работе [1] А. Гротендик показал, что для произвольного банахова пространства $X$ свойство аппроксимации эквивалентно однозначной определенности следа ядерного оператора. Это утверждение не переносится непосредственно на более общие классы пространств, поскольку здесь свойство аппроксимации перестает быть достаточным условием [2]. Тем не менее в модифицированном виде этот факт остается справедливым для любых локально выпуклых пространств [3]. Изучению условий однозначной определенности следа и близким вопросам посвящена серия работ Г. Л. Литвинова $[2-4]$.

В настоящей заметке предлагается решение проблемы однозначности следа для категории стереотипных локально выпуклых пространств, введенной автором в $[5,6]$. Мы отмечаем, что в этой категории однозначность следа эквивалентна естественно определяемому свойству стереотипной аппроксимации, а также анонсируем некоторые другие результаты, касающиеся свойства стереотипной аппроксимации и близких к нему условий.

1. Псевдополные и псевдонасыщенные пространства. В этом и следующем пунктах мы напомним некоторые определения работ $[5,6]^{1}$. Условимся морфизмом локально выпуклых пространств (ЛВП) называть произвольное линейное непрерывное отображение $\varphi: X \rightarrow Y$.

Множество $S$ в ЛВП $X$ мы, как обычно, называем вполне ограниченныл, если для всякой окрестности нуля $U$ в $X$ найдется конечное множество $F \subseteq X$,

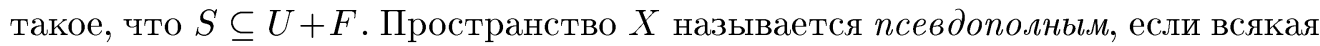
вполне ограниченная направленность Коши в $X$ сходится. Это равносильно тому, что всякое замкнутое вполне ограниченное множество в $X$ является компактом.

Как и в случае с полными пространствами, существует стандартная операция, позволяющая каждому ЛВП $X$ сопоставить ближайшее к нему «снаружи» псевдополное пространство $X^{\nabla}$, называемое его псевдопополнением.

ПРЕДЛОЖЕНИЕ 1. Каждому ЛВП $X$ можно поставить в соответствие морфизм $\nabla_{X}: X \rightarrow X^{\nabla}$ в некоторое псевдополное ЛВП $X^{\nabla}$ maк, итобы выполнялись следуюшие условия:

(i) $X$ псевдополно тогда и только тогда, когда $\nabla_{X}: X \rightarrow X^{\nabla}-$ изоморфизм;

(ii) для любого морфизма ЛВП $\varphi: X \rightarrow Y$ найдется единственный морфизм ЛВП $\varphi^{\nabla}: X^{\nabla} \rightarrow Y^{\nabla}$, замьккающий диаграмму

${ }^{1}$ Терминология и обозначения настоящей работы несколько отличаются от принятых в $[5,6]$, но все результаты сохраняют преемственность. В частности, $X^{\nabla}=\# X, X^{\Delta}={ }_{b} X$, $X^{\star}=X^{\prime}$. 


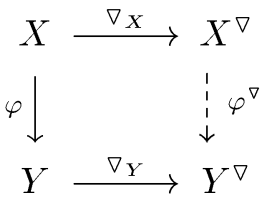

Множество $D$ в ЛВП $X$ мы называем емким, если для всякого вполне ограниченного множества $S \subseteq X$ найдется конечное множество $F \subseteq X$, такое, что $S \subseteq D+F$. Пространство $X$ мы называем псевдонасышенныл, если всякое замкнутое выпуклое уравновешенное емкое множество $D$ в $X$ является окрестностью нуля.

Оказывается, существует также стандартная конструкция, позволяющая каждому ЛВП $X$ сопоставить ближайшее к нему «изнутри» псевдонасышенное пространство $X^{\triangle}$, называемое его псевдонасыщением.

ПРЕДЛОЖЕНИЕ 2. Каждому ЛВП $X$ можно поставить в соответствие морфизм $\triangle_{X}: X^{\Delta} \rightarrow X$ из некоторого псевдонасышенного ЛВП $X^{\Delta}$ так, чтобы выполнялись следуюшие условия:

(i) $X$ псевдонасыиенно тогда и только тогда, когда $\triangle_{X}: X^{\Delta} \rightarrow X-$ изоморфизм;

(ii) для любого морфизма ЛВП $\varphi: Y \rightarrow X$ найдется единственньй мор$\oint$ фим ЛВП $\varphi^{\Delta}: Y^{\Delta} \rightarrow X^{\Delta}$, замыкающий диаграмму

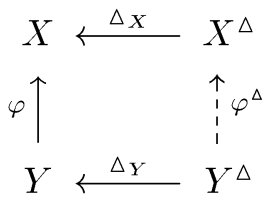

ПРЕДлОЖЕНИЕ 3. Если $X$ псевдонасыщенно, то псевдопополнение $X^{\nabla}$ также псевдонасыщенно, а если $X$ псевдополно, то псевдонасыщение $X^{\Delta}$ также псевдополно.

2. Стереотипные пространства. Для произвольного ЛВП $X$ над $\mathbb{C}$ обозначим символом $X^{\star}$ пространство линейных непрерывных функционалов $f: X \rightarrow \mathbb{C}$, наделенное топологией равномерной сходимости на вполне ограниченных множествах в $X$. Как уже отмечалось в работе [5], для ЛВП $X$ следующие условия эквивалентны:

(i) пространство $X$ псевдополно и псевдонасьиенно;

(ii) естественная инъекиия $i_{X}: X \rightarrow X^{\star \star}$ является изоморфизмом ЛВП.

Пространства $X$, удовлетворяющие этим условиям, мы называем стереотипныли. Класс $\mathfrak{S}$ стереотипных пространств весьма широк, поскольку, как отмечалось в [5], включает в себя все квазиполнье бочечные пространства. Он образует категорию с линейными непрерывными отображениями в качестве морфизмов. Эта категория предабелева и допускает любые индуктивные и проективные пределы.

Если $X$ и $Y$ - два стереотипных пространства, то пусть символ $Y \oslash X$ обозначает псевдонасыщение пространства морфизмов $\varphi: X \rightarrow Y$, наделенного топологией равномерной сходимости на вполне ограниченных множествах. Пространство $Y \oslash X$ стереотипно, и мы называем его (внутренним) пространством морфизмов из $X$ в $Y$. Переход к сопряженному отображению 
устанавливает изоморфизм стереотипных пространств

$$
Y \oslash X \cong X^{\star} \oslash Y^{\star} \text {. }
$$

Определим проективное стереотипное тензорное произведение $X \circledast Y$ и инъективное стереотипное тензорное произведение $X \odot Y$ стереотипных пространств $X$ и $Y$ формулами

$$
X \circledast Y=\left(X^{\star} \oslash Y\right)^{\star}, \quad X \odot Y=Y \oslash X^{\star} .
$$

Можно проверить, что для пространств Фреше $X \circledast Y$ есть обычное проективное тензорное произведение, а если, кроме того, $X$ обладает (классической) аппроксимацией, то $X \odot Y$ совпадает с обычным инъективным тензорным произведением (в смысле [7]). Для любых $x \in X$ и $y \in Y$ элементарный тензор $x \circledast y \in X \circledast Y$ и элементарный оператор $x \odot y \in X \odot Y$ определяются равенствами

$$
(x \circledast y)(\varphi)=\varphi(y)(x), \quad(x \odot y)(f)=f(x) y, \quad \varphi \in X^{\star} \oslash Y, f \in X^{\star} .
$$

В категории $\mathfrak{S}$ справедливы тождества

$$
\begin{array}{rlrl}
\mathbb{C} \circledast X & \cong X \cong X \circledast \mathbb{C}, & \mathbb{C} \odot X \cong X \cong X \odot \mathbb{C}, \\
(X \circledast Y) \circledast Z \cong X \circledast(Y \circledast Z), & (X \odot Y) \odot Z \cong X \odot(Y \odot Z), \\
X \circledast Y & \cong Y \circledast X, & X \odot Y \cong Y \odot X, \\
(X \circledast Y)^{\star} & \cong Y^{\star} \odot X^{\star}, & (X \odot Y)^{\star} \cong Y^{\star} \circledast X^{\star}, \\
X \oslash(Y \circledast Z) & \cong(X \oslash Y) \oslash Z, & (X \odot Y) \oslash Z \cong X \odot(Y \oslash Z),
\end{array}
$$

из которых, в частности, следует

ТеОРема 4. Категория $\mathfrak{S}$ стереотипных пространств является моноидальной категорией (в смьсле $[8,9])$ относительно каждой из операчий $\circledast$ $u \odot$, причем $\circledast$ задает на $\mathfrak{S}$ структуру замкнутой моноидальной категории c внутренним hот-функтором $\operatorname{Hom}(X, Y)=Y \oslash X$.

\section{3. Отображение Гротендика @ $X \circledast Y \rightarrow X \odot Y$ и свойство стерео- типной аппроксимации.}

Теорема 5. Для любых стереотипных пространств $X$ и $Y$ существует единственный морфизм стереотипных пространств

$$
\text { @ : } X \circledast Y \rightarrow X \odot Y \text {, }
$$

называемый отображением Гротендика, который удовлетворяет тождеству

$$
@(x \circledast y)=x \odot y .
$$

Пусть символ $\mathscr{L}(X)$ обозначает внутреннее пространство эндоморфизмов: $\mathscr{L}(X)=X \oslash X$. Очевидно, что

$$
\mathscr{L}(X)=X^{\star} \odot X, \quad \mathscr{L}(X)^{\star}=X^{\star} \circledast X .
$$

Условимся говорить, что стереотипное пространство $X$ обладает стереотипной аппроксимачией, если единичный оператор $1_{X}$ приближается конечномерными в пространстве $\mathscr{L}(X)$. Это означает, что множество $\mathscr{F}(X)$ конечномерных операторов плотно в $\mathscr{L}(X)$, т.е. выполняется равенство $\overline{\mathscr{F}(X)}=$ $\mathscr{L}(X)$. Понятно, что стереотипная аппроксимация влечет за собой обычную 
аппроксимацию. Отсюда следует, что существуют пространства без стереотипной аппроксимации. С другой стороны, нетрудно предъявить простейшие достаточные условия для стереотипной аппроксимации. Таким условием будет существование базиса в пространстве $X$ (см. п. 5 ниже).

Теорема 6. Для стереотипного пространства $X$ следуюшие условия эквивалентнь:

(i) $X$ обладает стереотипной аппроксимацией;

(ii) $X^{\star}$ обладает стереотипной аппроксимацией;

(iii) отображение Гротендика @ : $\mathscr{L}(X)^{\star}=X^{\star} \circledast X \rightarrow X^{\star} \odot X=\mathscr{L}(X)$ является мономорфизмом;

(iv) отображение Гротендика@: $\mathscr{L}(X)^{\star}=X^{\star} \circledast X \rightarrow X^{\star} \odot X=\mathscr{L}(X)$ являетсл эпиморфизмом;

(v) для всякого стереотипного пространства $Y$ отображение Гротендика@: $Y \circledast X \rightarrow Y \odot X$ является мономорфизмом;

(vi) для всякого стереотипного пространства $Y$ отображение Гротенди$к а @: Y \circledast X \rightarrow Y \odot X$ является эпиморфизмом.

Можно проверить, что стереотипная аппроксимачия наследуется прямьми произведениями и прямыми суммами. По аналогии с известным контрпримером Шанковского для банаховой теории [10] можно было бы ожидать, что стереотипная аппроксимация не наследуется пространствами операторов. Удивительно, что стереотипная теория в этом смысле оказывается устроенной проще:

ТЕОРема 7. Пусть $X$ и $Y$ - cmереотипные пространства со стереотипной аппроксимаиией. Тогда пространства $X \oslash Y, X \circledast Y, X \odot Y$ также обладают стереотипной аппроксимачией.

4. Проблема однозначности следа. Определим на тензорном произведении $X^{\star} \circledast X=\mathscr{L}(X)^{\star} \oint$ ункиионал свертки формулой

$$
\text { cont }: \mathscr{L}(X)^{\star} \rightarrow \mathbb{C}, \quad \operatorname{cont}(\alpha)=\alpha\left(1_{X}\right) .
$$

Поскольку на элементарных тензорах он, очевидно, действует по формуле

$$
\operatorname{cont}(f \circledast x)=f(x),
$$

этот функционал будет (единственным по теореме 11 из [5]) непрерывным продолжением на $X^{\star} \circledast X$ билинейной формы $(f, x) \mapsto f(x)$.

Условимся говорить, что в $X$ корректно определен след, если ядро Ker @ отображения Гротендика @: $\mathscr{L}(X)^{\star} \rightarrow \mathscr{L}(X)$ содержится в ядре $\operatorname{Ker}($ cont) функционала свертки

$$
\operatorname{Ker} @ \subseteq \operatorname{Ker}(\text { cont }) .
$$

Ясно, что в этом случае свертка, рассматриваемая как функционал на конечномерных операторах, однозначно продолжается до функиионала следа tr на всех операторах, являющихся образами тензоров из $X^{\star} \circledast X$ при отображении Гротендика.

ТЕОрема 8. Для стереотипного пространства $X$ следуюшие условия эквивалентнь:

(i) X обладает стереотипной аппроксимацией;

(ii) в $X$ корректно определен след. 
5. Пространства с базисом. Пусть $I$ - произвольное множество индексов и $2_{I}$ - система всех его конечных подмножеств. Определим на $2_{I}$ частичный порядок теоретико-множественным включением $A \subseteq B$. Назовем $n р а в и-$ лом суммирования на множестве $I$ произвольную конфинальную подсистему $\mathscr{I} \subseteq 2_{I}$ (для любого $A \in 2_{I}$ сушествует $F \in \mathscr{I}$, такое, что $A \subseteq F$ ). Семейство $\left\{x_{i} ; i \in I\right\}$ элементов ЛВП $X$ условимся называть суммируемьм по правилу $\mathscr{I}$, если существует элемент $x \in X$, такой, что для любой окрестности нуля $U \subseteq X$ существует $C \in \mathscr{I}$, такое, что для любого $F \in \mathscr{I}$ включение $C \subseteq F$ влечет за собой включение $\sum_{i \in F} x_{i}-x \in U$. При этом $x$ будет называться суммой семейства $\left\{x_{i} ; i \in I\right\}$ по правилу $\mathscr{I}$ и обозначаться $x=\sum_{i \in I} x_{i} \quad(\mathscr{I})$.

Семейство пар $\left\{\left(e_{i}, e_{i}^{\prime}\right) ; i \in I\right\}, e_{i} \in X, e_{i}^{\prime} \in X^{\star}$, назовем базисом стереотипного пространства $X$ относительно $\mathscr{I}$, если оно удовлетворяет условиям

$$
\left\langle e_{i}, e_{j}^{\prime}\right\rangle=\left\{\begin{array}{ll}
0, & i \neq j, \\
1, & i=j,
\end{array} \quad \sum_{i \in I} e_{i}^{\prime} \odot e_{i}=1_{X} \quad(\mathscr{I}) .\right.
$$

При подходящем выборе правила суммирования $\mathscr{I} \subseteq 2_{I}$ наше определение становится эквивалентным классическим определениям базиса. Например, если $I=\mathbb{N}$, а $\mathscr{I}$ состоит из множеств вида $F=\{1, \ldots, n\}, n \in \mathbb{N}$, то для бочечных пространств понятие базиса относительно $\mathscr{I}$ совпадает с понятием базиса Шаудера [11]. Если же $I=\mathbb{N}$ и $\mathscr{I}=2_{I}$, то мы получаем определение безусловного базиса [11]. Примерами пространств с несчетными базисами $\left(\right.$ при $\mathscr{I}=2_{I}$ ) являются пространства $\mathbb{C}^{I}$ и $\mathbb{C}_{I}$ (рассматривавшиеся автором в $[12]$, с точностью до замены $\mathbb{C}$ на $\mathbb{R})$. Как уже говорилось, существование базиса (относительно произвольного правила суммирования) является достаточнылм условием для стереотипной аппроксимаиии. Отметим, что $X$ обладает базисом тогда и только тогда, когда $X^{\star}$ обладает базисом. Из очевидной формулы $\operatorname{cont}(u)=\sum_{i \in I} e_{i}^{\prime}\left(u\left(e_{i}\right)\right), u \in \mathscr{L}(X)^{\star}$, следует, что на пространстве с базисом след, определенный выше, совпадает с матричным следом (т. е. является суммой диагональных элементов).

Правило суммирования $\mathscr{I}$ назовем замкнутылм, если $F \cup G \in \mathscr{I}$ для любых $F, G \in \mathscr{I}$. Как и в случае со свойством аппроксимации, свойство иметь базис относительно замкнутого правила суммирования наследуется прямьми произведениями и прямыми суммами. Кроме того, справедлива

ТЕОрема 9. Если стереотипные пространства X и ми относительно замкнутьх правил суммирования, то пространства $Y \oslash X$, $X \circledast Y, X \odot Y$ тоже обладают базисами.

В частности, в противоположность результату Шанковского [10], если $X$ стереотипное пространство с базисом относительно замкнутого правила суммирования (например, гильбертово), то $\mathscr{L}(X)$ - тоже стереотипное пространство с базисом.

\section{ЛитеРАТУРА}

1. Grothendieck A. Mem. Amer. Math. Soc., 16, 1955. 2. Литвинов Г. Л. В кн.: Теория функций, функциональный анализ и их приложения, т. 39, 1983, с. 73-87; англ. вариант: Selecta Matematica Sovietica, 11, No. 1, 25-40 (1992). 3. Литвинов Г. Л., Ломоносов В. И. В кн.: Труды семинара по векторному и тензорному анализу, т. 20, 1981, с. 210-227; англ. вариант: Selecta Matematica Sovietica, 8, No. 4, 323-339 (1989). 
4. Литвинов Г. Л. В кн.: Труды семинара по векторному и тензорному анализу, т. 19, 263-272 (1979); англ. вариант: Selecta Matematica Sovietica, 8, No. 3, 203-212 (1989). 5. Акбаров С. С. Матем. заметки, 57, № 3, 463-466 (1995). 6. Акбаров С. С. Функц. анализ и его прил., 29, вып. 4, 68-72 (1995). 7. Jarchow H. Locally convex spaces. B. G. Teubner, Stuttgart, 1981. 8. MacLane S. Categories for working mathematician. Springer, N. Y., 1971. 9. Артамонов В. А., Салий В. Н., Скорняков Л. А., Шеврин Л. Н., Шульгейфер Е. Г. Общая алгебра. Наука, М., 1991. 10. Szankowski A. Acta Math., 147, 89-108 (1981). 11. Шефер X. Топологические векторные пространства. Мир, М., 1971. 12. Акбаров С. С. Известия РАН. 59, № 1, 3-48 (1995).

Московский государственный

технический университет им. Н. Э. Баумана

Поступило в редакцию e-mail: serge@mx.bmstu.ru

9 декабря 1997 г.

УДК 517.982.27

\section{Кратные ряды Радемахера в симметричных пространствах}

(c) 1999. С. В. Асташкин

Пусть

$$
r_{k}(t)=\operatorname{sign} \sin 2^{k-1} \pi t \quad(k=1,2, \ldots)
$$

- система функций Радемахера на $I=[0,1]$. Рассмотрим множество функций $x(t)$, представимых в виде

$$
x(t)=\sum_{i \neq j} a_{i, j} r_{i}(t) r_{j}(t) \quad(t \in I) .
$$

Как и в случае рядов по системе Радемахера (см., например, [1, с. 148-150]), ряд (1) сходится почти всюду на $I$ (т. е. существует предел его прямоугольных частичных сумм) тогда и только тогда, когда последовательность коэффициентов $a=\left(a_{i, j}\right)_{i \neq j}$ принадлежит $l_{2}$.

Ортонормированная на $I$ система $\left\{r_{i} r_{j}\right\}_{i \neq j}$, в отличие от системы Радемахера, состоит из функций, не являющихся независимыми. Тем не менее ее свойства во многих отношениях напоминают свойства семейств независимых и равномерно ограниченных функций. Так, например, условие $\sum_{i \neq j} a_{i, j}^{2}<$ $\infty$ влечет за собой суммируемость функции $\exp (\alpha|x(t)|)$ для любого $\alpha>0$ $[2$, p. 105]. В то же время есть и существенные отличия. Например, кратная система $\left\{r_{i} r_{j}\right\}_{i \neq j}$ не является системой Сидона [3]. Для более полного изучения ее свойств (в частности, вопроса о возможности усиления утверждения об экспоненциальной суммируемости) целесообразно перейти к общим симметричным пространствам.

Напомним, что банахово пространство $X$ измеримых по Лебегу на $I$ функций $x=x(t)$ называется симметричным пространством (СП), если из неравенства $x^{*}(t) \leqslant y^{*}(t)\left(z^{*}(t)\right.$ - невозрастающая перестановка функции $|z(u)|$ $[4$, c. 83]) и того, что $y \in X$, следует, что $x \in X$ и $\|x\| \leqslant\|y\|$.

Кроме пространств $L_{p}(1 \leqslant p \leqslant \infty)$, важными примерами СП являются пространства Орлича и Марцинкевича. 